\title{
Aplicación de Fuzzy-AHP y COPRAS en la selección de la mejor alternativa para el maquinado a alta velocidad de estructuras delgadas en aleaciones de aluminio Al 5083
}

\section{Application of Fuzzy-AHP and COPRAS in the selection of the best alternative for high speed machining of thin structures in Al 5083 aluminum alloys}

\author{
Hiovanis Castillo Pantoja 1 https://orcid.org/0000-0003-0091-0904, Ángel Infante Haynes ${ }^{1}$ \\ https://orcid.org/0000-0002-6462-5339, Roberto Pérez Rodríguez 1 https://orcid.org/0000-0001-5741-5168, \\ Ricardo Lorenzo Ávila Rondón ${ }^{2}$ https://orcid.org/0000-0001-6730-5789 \\ ${ }^{1}$ Universidad de Holguín. Facultad de Ingeniería, Holguín, Cuba \\ sotosilva74@gmail.com, ehaynes@uho.edu.cu, \\ roberto.perezeuho.edu.cu
}

${ }^{2}$ Universidad Autónoma de Coahuila. Coahuila, México rlar001@yahoo.com

\section{(c) () (9)}

Esta obra está bajo una licencia internacional Creative Commons Atribución-NoComercial 4.0.
Enviado: $\quad 2021 / 07 / 05$

Aceptado: $\quad 2021 / 09 / 28$

Publicado: $\quad 2021 / 11 / 30$

\section{Resumen}

En la siguiente investigación se muestra una metodología que combina el método multicriterio COPRAS y el de inteligencia artificial AHP Difuso, que busca mejorar la toma de decisiones dentro de los procesos de planeación en los talleres de maquinado. El primero de los métodos permite determinar el criterio de mayor importancia a cumplir como exigencia en la fabricación; el segundo método, busca la selección de la mejor alternativa, con los valores para el maquinado a alta velocidad que permita fabricar la pieza rectangular de aleación de aluminio 5083. Para el análisis multicriterio los parámetros seleccionados en el proceso de maquinado de piezas de aluminio de estructura delgada son: la rugosidad superficial y la deformación de la pieza. Al aplicar el método Fuzzy-AHP, se determina que el criterio de mayor peso lo constituye la deformación de la pieza en la estructura delgada. Con la evaluación de los criterios, se aplica COPRAS y el resultado del índice de utilidad determina que la alternativa tres es la mejor, por tanto, al implementar los parámetros de entrada: $\mathrm{S}=15000 \mathrm{rpm}, \mathrm{doc}=0.30$ $\mathrm{mm}, \mathrm{ts}=7.0 \mathrm{~mm}, \mathrm{~F}=9000 \mathrm{~m} / \mathrm{min}$, se garantiza la calidad en la superficie y baja deformación de la pieza. Se concluye que la metodología de Fuzzy-AHP y COPRAS resulta una excelente

Sumario: Introducción, Materiales y Métodos, Resultados y Discusión y Conclusiones.

Como citar: Castillo, H., Infante, A., Pérez, R. \& Ávila, L. (2021). Aplicación de Fuzzy-AHP y COPRAS en la selección de la mejor alternativa para el maquinado a alta velocidad de estructuras delgadas en aleaciones de aluminio Al 5083. Revista Tecnológica - Espol, 33(2), 109-121.

http://www.rte.espol.edu.ec/index.php/tecnologica/article/view/836 
herramienta, con un bajo costo y buena fiabilidad, como solución a aplicar en los talleres de maquinado para mejorar la toma de decisiones en la planeación de procesos.

Palabras clave: AHP Difuso, COPRAS, maquinado a alta velocidad, inteligencia artificial.

\begin{abstract}
The following research shows a methodology that combines the multi-criteria method COPRAS and the artificial intelligence method AHP Fuzzy, which seeks to improve decision making within the planning processes in machining shops. The first method allows us to determine the most important criterion to be fulfilled as a manufacturing requirement; the second method seeks the selection of the best alternative, with the values for high speed machining that will allow the manufacturing of the rectangular piece of aluminum alloy 5083. For the multi-criteria analysis, the parameters selected for the machining process of thinstructured aluminum parts are: surface roughness and part deformation. By applying the FuzzyAHP method, it is determined that the most important criterion is the deformation of the part in the thin structure. With the evaluation of the criteria, COPRAS was applied and the result of the utility index determined that alternative three is the best, therefore, by implementing the input parameters: $\mathrm{S}=15000 \mathrm{rpm}, \mathrm{doc}=0.30 \mathrm{~mm}, \mathrm{ts}=7.0 \mathrm{~mm}, \mathrm{~F}=9000 \mathrm{~m} / \mathrm{min}$, surface quality and low deformation of the part is guaranteed. We conclude that the Fuzzy-AHP and COPRAS methodology is an excellent tool, with low cost and good reliability, as a solution to be applied in machine shops to improve decision making in process planning.
\end{abstract}

Keywords: COPRAS, AHP Fuzzy, high speed machining, artificial intelligent.

\title{
Introducción
}

El fresado constituye uno de los procesos tecnológicos con más utilización en la industria contemporánea y el material de aluminio, por sus características físico-mecánicas, es el más relevante al construir piezas de estructura delgada. Es por ello, que ante la competencia productiva se necesita la introducción de equipamiento tecnológico especial para alcanzar las grandes metas productivas deseadas; este es el caso del fresado a alta velocidad, que permite disminuir un número especial de máquinas herramientas. Dentro de este proceso de fabricación las principales dificultades que se aprecian son relacionados con: la calidad de rugosidad superficial y las deformaciones en las piezas. Estas problemáticas están en gran medida relacionadas dentro de la planeación de procesos con la selección correcta de los parámetros de maquinado.

En este contexto, para dar solución a esta problemática, los ingenieros y especialistas buscan nuevos métodos que le proporcionen estrategias; es ahí donde el avance de la inteligencia artificial le ha abierto un extraordinario campo. Entre las soluciones más empleadas está la selección óptima de los parámetros de maquinado, desde el enfoque tradicional, hasta el novedoso y útil análisis multicriterios.

Para el caso de la fabricación con material de aleaciones de aluminio de piezas con estructura delgada, los expertos han definidos tres criterios con una marcada influencia, estos son: la rugosidad superficial en la dirección del avance, de la dirección transversal y la deformación de la pieza. El valor de los parámetros al fabricar las piezas puede dar como resultado varias alternativas, por lo que se define como objetivo, determinar la solución más óptima con inteligencia artificial: Método Fuzzy-AHP para definir el criterio de mayor validez y COPRAS para determinar la jerarquía de las soluciones. 
En otro orden, (Paker et al., 2018), estudian la relevancia que tienen los procesos de manufactura y el diseño CAD enfocado en la industria del automovilismo y logran establecer, a través de métodos analíticos de jerarquía (AHP), los principales criterios que permitan alcanzar las mejores modificaciones de los diseños creados en cada uno de los proyectos para luego realizar su fabricación. (Bhowmik et al., 2019) revisa en los últimos 15 años varios de los métodos multicriterios para la toma de decisiones (MCDM), en los que se emplea la optimización de los procesos de manufactura.

Dentro de los autores que han utilizado los modelos de inteligencia artificial, FuzzyAHP están (Vukman et al., 2019), que aplica la lógica difusa en el fresado de piezas de estructuras delgadas, comprueba parámetros de corte y su influencia en el performance de la superficie de acabado de las piezas. En el conformado de chapas de metal, se tiene en cuenta las terminaciones de calidad a la hora de obtener las diferentes piezas. (Bhowmik et al., 2019) demuestra con la utilización del método COPRAS agrupa 4 criterios en su modelo, para mejorar los efectos mecánicos en el máximo adelgazamiento de las chapas y garantizar la calidad de las producciones.

En la industria actual de la fabricación de piezas por procesos de torneado a partir de los centros de maquinado con CNC, en su investigación (Patil \& Kothavale, 2020) explican la prioridad que se establece a través del análisis jerárquico (AHP) que parte de cuatro criterios (SS, CNC, XZAS, TS, EES, and MT) como los más críticos para definir las estrategias correctas, siendo de gran utilidad en beneficiar los procesos de gestión de mantenimiento de estas máquinas herramientas. Durante la evolución de los procesos de fabricación modernos los sistemas de fabricación flexible, juegan un importante papel en cada uno de los talleres de maquinado (Patil \& Kothavale, 2020) se encargan de presentar una investigación donde recorren las diferentes técnicas de modelados: matemática, inteligencia artificial, toma de decisiones a partir del análisis multicriterios y jerárquicos, redes de Petri y la simulación, resaltando finalmente la importancia de estos en los procesos de fabricación. (Patil \& Kothavale, 2020) también proponen el framework Fuzzy-AHP y PROMETHEE para en análisis multicriterio, que busca a partir de 22 soluciones la mejor alternativa que permita disminuir la reducción de residuos en los procesos de manufactura. Señalan la utilidad de los métodos MCDM al buscar la mejor alternativa de los parámetros que garantizan las condiciones óptimas de calidad en la manufactura de orificios. Como criterios se avalúa (redondes, tamaño y conicidad), tomando como variables del análisis: el espesor de la pieza de trabajo, perfil de la herramienta, material y tipo, entre otros. El modelo se ajusta con los métodos MCDM de ARAS-TOPSIS. A partir de los prácticas tradicionales de maquinado la sostenibilidad es una temática importante en las empresas de manufactura, y en esencia en la toma de decisiones (Patil \& Kothavale, 2020) en su investigación proponen una combinación de dos métodos MCDM: WESPAS-SECA e incorporan, para mejorar el mismo, la combinación de 2-fuzzy (IT2FSs) en su modelo para optimizar el análisis de incertidumbre y, de esta forma, realiza una mejor evaluación de las estrategias sostenibles. (Sen et al., 2020), en el correcto uso de líquido de refrigeración integración de los métodos Fuzzy AHP-ARAS. (Jasiulewicz-Kaczmarek et al., 2021), en la selección de estrategias de mantenimiento, analiza los procesos de fabricación sostenible desde el punto de vista de la evaluación de los factores de impacto en los procesos de mantenimiento, se aplica F-AHP para determinar la jerarquía y pesos relativos y con F-TOPSIS se demuestra su factibilidad para determinar la mejor solución y seleccionar los factores de mantenimiento más importantes que tienen un impacto en los procesos de fabricación sostenibles.

Como se puede apreciar son varios los trabajos que en su desarrollo, de una manera simple o combinado, han utilizado análisis jerárquico de proceso y método multicriterio en la 
toma de decisiones para los procesos de maquinado. Sin embrago, en la literatura no se aprecian trabajos referidos a la combinación de análisis de incertidumbre y multicriterio para el maquinado a alta velocidad en piezas de estructuras delgadas de aluminio, por lo que resulta una novedad su aplicación.

\section{Caso de estudio}

\section{Materiales y Métodos}

Las operaciones de fresado de alta velocidad se realizaron en Quick Centro de mecanizado Jet AV1612, equipado con HEI-Sistema CNC de DENHAIN para un control preciso del mecanizado con una velocidad máxima de husillo de $20.000 \mathrm{rpm}$ y velocidad de alimentación de $25 \mathrm{~m} / \mathrm{min}$. Se seleccionó la pieza de trabajo para el experimento de una aleación de Al 5083 en forma rectangular con medidas de $140 \mathrm{~mm} \times 70 \mathrm{~mm} \times 5 \mathrm{~mm}$. La pieza de trabajo fue montada en un accesorio especial aplicando 6 pernos, además sujeta en la cama de la máquina herramienta. La composición química y las propiedades físicas del material de la pieza de trabajo son recogidas en la Tabla 1 y Tabla 2 respectivamente.

\section{Tabla 1}

Composición Química de la Aleación de Aluminio 5083

\begin{tabular}{|l|l|}
\hline Elemento & \% Presente \\
\hline $\mathrm{Si}$ & 0.4 \\
\hline $\mathrm{Fe}$ & 0.4 \\
\hline $\mathrm{Cu}$ & 0.1 \\
\hline $\mathrm{Mn}$ & $0.4-1.0$ \\
\hline $\mathrm{Mg}$ & $4.0-4.9$ \\
\hline $\mathrm{Zn}$ & 0.25 \\
\hline $\mathrm{Ti}$ & 0.15 \\
\hline $\mathrm{Cr}$ & $0.05-0.25$ \\
\hline $\mathrm{Al}$ & Balance \\
\hline
\end{tabular}

Tabla 2

Propiedades Físicas de la Aleación de Aluminio 5083

\begin{tabular}{|l|l|}
\hline \multicolumn{1}{|c|}{ Propiedades } & \multicolumn{1}{c|}{ Valor } \\
\hline Density & $2650 \mathrm{~kg} / \mathrm{m}^{3}$ \\
\hline Melting point & $570^{\circ} \mathrm{C}$ \\
\hline Modulus of elasticity & $72 \mathrm{GPa}$ \\
\hline Electrical resistivity & $0.058 \times 10^{-6} \Omega-\mathrm{m}$ \\
\hline Thermal conductivity & $121 \mathrm{~W} / \mathrm{m}-\mathrm{K}$ \\
\hline Thermal expansion & $25 \times 10^{-6} / \mathrm{K}$ \\
\hline
\end{tabular}

\section{Tabla 3}

Propiedades Mecánicas de la Aleación de Aluminio 5083

\begin{tabular}{|l|c|c|}
\hline \multicolumn{1}{|c|}{ Temper } & H32 & 0/HIII \\
\hline Proof stress 0.2 \% (MPa) & 240 & 145 \\
\hline Tensile strength & 330 & 300 \\
\hline Shear strength (MPa) & 185 & 175 \\
\hline Elongation A5 (\%) & 17 & 23 \\
\hline Hardness Vickers & 95 & 75 \\
\hline
\end{tabular}




\section{Fuzzy AHP}

El proceso analítico jerárquico se ha convertido en una herramienta utilizada para diferentes investigaciones. Dentro de los métodos de la toma de decisiones Fuzzy AHP ayuda a establecer de manera cualitativa y cuantitativa las prioridades en vista a solucionar los diferentes problemas. En su desarrollo se puede determinar las mínimas acciones de evaluación a los criterios complejos. Sin embargo, a la hora de analizar las posibilidades de tomar decisiones existen incertidumbres y se hace difícil establecer valores numéricos al dar las respuestas. AHP se convierte en un método muy idóneo para que los usuarios se ocupen de la vaguedad o incertidumbre en la toma de decisiones de los procesos (Mehdi Ajalli, 2017).

FAHP se fundamenta a partir de una prioridad local con una porción de preferencia que combinado se genera, lo que se conoce como las prioridades globales. Las prioridades Fuzzy en el cálculo FAHP se basan en operaciones aritméticas para valores trapezoidales $o$ triangulares. Sin embargo, a pesar de su alto uso, los críticos analizan su grado de consistencia, que está dado porque no existe la articulación específica de reconciliación entre la matriz de comparación y el empleo de la información, la probabilidad de errores al establecer el nivel de prioridad y la carencia de un mecanismo para eliminar la inconsistencia de los datos (Mehdi Ajalli, 2017). Para la solución del problema de selección de alternativas se emplea el Método Chang, en el que cada objeto es tomado y se extiende su análisis por cada meta analizada respectivamente.

La escala lingüística es empleada para realizar las comparaciones, reflexión de contenido impreciso debido a la incertidumbre que en ocasiones ocurre a partir de los criterios individuales o las variaciones en la percepción de los analistas (Kaori Ota, 2008). Para esta investigación se toma la tabla lingüística para una escala Fuzzy triangular, que aparece en la Tabla 4.

\section{Tabla 4}

Escala Lingüística para Nivel de Importancia en el Grado Fuzzy Triangular

\begin{tabular}{|r|l|c|}
\hline 1 & Igual & $(1,1,1)$ \\
\hline 3 & Moderado & $(2,3,4)$ \\
\hline 5 & Fuerte & $(4,5,6)$ \\
\hline 7 & Muy Fuerte & $(6,7,8)$ \\
\hline 9 & Extremadamente fuerte & $(9,9,9)$ \\
\hline \multirow{3}{*}{110} & \multirow{3}{*}{ Valores Intermedios } & $(1,2,3)$ \\
\cline { 3 - 3 } & & $(3,4,5)$ \\
\hline & & $(5,6,7)$ \\
\cline { 3 - 3 } & & $(7,8,9)$ \\
\hline
\end{tabular}

Paso 1. El valor fuzzy sintético extendido con respecto al objeto $i_{\text {th }}$ es determinado por:

$$
S i=\sum_{j=1}^{m} M_{g i}^{j} \oplus\left[\sum_{i=1}^{n} \sum_{j=1}^{m} M_{g i}^{j}\right]^{-1}
$$

Para establecer $\sum_{j=1}^{m} M_{g i}^{j}$, la operación de adición fuzzy del valor de análisis extendido $\mathrm{m}$ por la matriz determinada es realizado por:

$$
\sum_{\mathrm{j}=1}^{\mathrm{m}} \mathrm{M}_{\mathrm{gi}}^{\mathrm{j}}=\left(\sum_{j=1}^{m} l_{j}, \sum_{j=1}^{m} m_{j}, \sum_{j=1}^{m} u_{j}\right)
$$


Y para obtener $\left[\sum_{i=1}^{n} \sum_{j=1}^{m} M_{g i}^{j}\right]$, por la realización de la operación suma de fuzzy de $M_{g i}^{j}(=1,2, \ldots, m)$ como

$$
\left[\sum_{i=1}^{n} \sum_{j=1}^{m} M_{g i}^{j}\right]^{-1}=\left(\sum_{j=1}^{m} l_{j}, \sum_{j=1}^{m} m_{j}, \sum_{j=1}^{m} u_{j}\right)
$$

$\mathrm{Y}\left[\sum_{i=1}^{n} \sum_{j=1}^{m} M_{g i}^{j}\right]^{-1}$ puede ser calculado por la inversa de la Ecuación 4 como sigue:

$$
\left[\sum_{i=1}^{n} \sum_{j=1}^{m} M_{g i}^{j}\right]^{-1}=\left(\frac{1}{\sum_{i=1}^{n} U_{i}}, \frac{1}{\sum_{i=1}^{n} m_{i}}, \frac{1}{\sum_{i=1}^{n} l_{i}}\right)
$$

Paso 2. Como $M_{1}=\left(l_{1}, m_{1}, u_{1}\right)$ y $M_{2}=\left(l_{2}, m_{2}, u_{2}\right)$ son dos números triangulares fuzzy, la mínima posibilidad de $M_{1} \geq M_{2}$ es definico como:

$$
V=\left(M_{2} \geq M_{1}\right)=\sup \left[\min \left(\mu_{M 1}(x), \mu_{M 2}(y)\right)\right] y \geq x
$$

Y puede ser expresado de la forma siguiente:

$$
V\left(M_{2} \geq M_{1}\right)=h g\left(M_{1} \cap M_{2}\right)=\mu_{M 2}(d)=\left\{\begin{array}{c}
1 \text { if } m_{2} \geq m_{1} \\
0 \text { if } l_{2} \geq u_{2} \\
\left(l_{1}-u_{2}\right)
\end{array}\right]
$$

Donde $d$, como se muestra en la Figura 1, es la ordenada del punto de intersección alto $\mathrm{D}$, entre $\mu_{\mathrm{m} 1 \mathrm{y}} \mu_{\mathrm{m} 2}$. Para comparar $M_{1}=\left(l_{1}, m_{1}, u_{1}\right)$ y $M_{2}=\left(l_{2}, m_{2}, u_{2}\right)$, se necesita de los dos valores $\mathrm{V}\left(\mathrm{M}_{1} \geq \mathrm{M}_{2}\right)$ y $\mathrm{V}\left(\mathrm{M}_{2} \geq \mathrm{M}_{1}\right)$.

\section{Figura 1}

Intersección entre M1 y M2

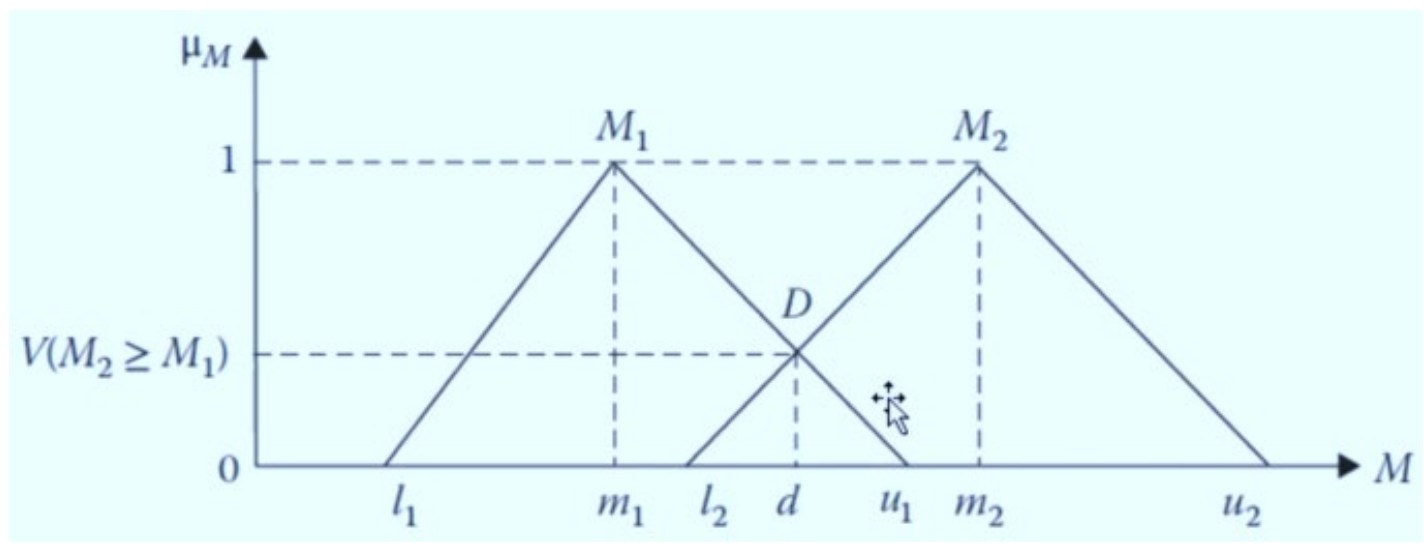

Paso 3. La mínima posibilidad para el número convexo fuzzy es mayor que fuzzy convexo $\mathrm{k}, \mathrm{Mi}(\mathrm{i}=1,2, \ldots \mathrm{k})$ los valores pueden ser definidos por:

$\mathrm{V}\left(\mathrm{M} \geq M_{1}, M_{2}, M_{k}\right)=v\left[\geq M_{1} y M \geq M_{2} y M \geq M_{k}\right]=\operatorname{minv} M \geq M_{1}, i=1,2 \ldots, k$

Se asume que d' $\left(A_{i}\right)=\min \left(S_{i} \geq S_{k}\right)$ f ó $k=1,2, \ldots n ; k \neq i$, entonces el peso del vector se obtiene por: 


$$
W^{\prime}=\left(d^{\prime}\left(A_{1}\right), d^{\prime}\left(A_{2}^{\prime} \ldots, d^{\prime}\left(A_{n}\right)\right)^{T}\right.
$$

Donde Ai $(\mathrm{i}=1,2, \ldots \mathrm{n})$ son $\mathrm{n}$ elementos.

Paso 4. Normalización, los vectores de pesos normalizados son:

$$
W=\left(d\left(A_{1}\right), d\left(A_{2}\right), d\left(A_{n}\right)\right)^{T}
$$

Donde W es un número no fuzzy.

\section{COPRAS (COmplex PRoportional ASessment)}

El método COPRAS (Turskis, 2008), selecciona las mejores alternativas de decisión considerando las soluciones ideales y las peores-ideales, en una clasificación y evaluación paso a paso de las alternativas en términos de su importancia y grado de utilidad. El algoritmo del método COPRAS consta de los siguientes pasos:

Paso 1. Desarrollo de la matriz inicial de decisión

$$
X=\left[X_{i j}\right]_{m x n}=\left[\begin{array}{cccc}
X_{11} & X_{12} & \ldots & X_{1 n} \\
X_{21} & X_{22} & \ldots & X_{2 n} \\
\ldots & \ldots & & \ldots \\
X_{m 1} & X_{m 2} & \ldots & X_{m n}
\end{array}\right]
$$

Donde $\mathrm{m}$ es el número de alternativas y n número de criterios.

Paso 2: Cálculo de la matriz de decisión normalizada (Ighravwe \& Oke, 2020)

$$
\mathrm{R}=R=\left[r_{i j}\right]_{m x n}=\frac{X_{i j}}{\sum_{i=1}^{m} X_{i j}}
$$

Paso 3: Determinar pesos de la matriz de decisión normalizada

$$
D=\left[y_{i j}\right]_{m x n}=r_{i j} X W_{j} i=1,2, \ldots, m y j=1,2, \ldots, n
$$

Paso 4: Las suma pesos normalizados de la matriz de decisión

$$
\begin{aligned}
& S_{+i}=\sum_{j=1}^{n} y_{+i j} \\
& S_{-i}=\sum_{j=1}^{n} y_{-i j}
\end{aligned}
$$

Se separan las sumas de los atributos beneficiosos y no beneficiosos.

Paso 5: Determinar la importancia relativa de las alternativas

$$
Q_{i}=S_{+i}+\frac{S_{-\min } \sum_{i=1}^{m} S_{-i}}{S_{-i} \sum_{i=1}^{m}\left(S_{-\min } / S_{-i}\right)} \quad(i=1,2, \ldots m)
$$

La importancia relativa $\mathrm{Q}_{i}$ de una alternativa muestra el grado de satisfacción alcanzada por esta alternativa. 
Paso 6: Cálculo del índice de la utilidad cuantitativa

$$
U_{i}=\left[\frac{Q_{i}}{Q_{\max }}\right] x 100 \%
$$

Tabla 5

Plan de valores experimentales y resultados de salida

\begin{tabular}{|c|c|c|c|c|c|c|c|}
\hline \multirow{2}{*}{ NNo. } & \multicolumn{3}{|c|}{ Parámetros de corte } & \multicolumn{3}{c|}{ Resultados } \\
\cline { 2 - 8 } & S(rpm) & doc(mm) & ts(mm) & $\mathbf{F}(\mathbf{m} / \mathbf{m i n})$ & Ra-Fd( $\boldsymbol{\mu m})$ & Ra-Td( $\boldsymbol{\mu m})$ & TWD \\
\hline 1 & 12.431 & 0.21 & 4.00 & 3600 & 4.583 & 3.952 & 0.060 \\
\hline 2 & 14.490 & 0.22 & 5.26 & 6668 & 5.457 & 4.742 & 0.066 \\
\hline 3 & 15.000 & 0.30 & 7.00 & 9000 & 6.540 & 4.979 & 0.105 \\
\hline 4 & 12.840 & 0.10 & 4.96 & 9000 & 5.716 & 5.169 & 0.059 \\
\hline 5 & 11.935 & 0.15 & 3.00 & 8892 & 5.394 & 5.527 & 0.060 \\
\hline 6 & 12.780 & 0.30 & 3.00 & 6192 & 5.356 & 5.275 & 0.100 \\
\hline 7 & 9000 & 0.10 & 3.00 & 8055 & 5.174 & 5.432 & 0.064 \\
\hline 8 & 9000 & 0.30 & 3.00 & 9000 & 5.833 & 6.264 & 0.084 \\
\hline 9 & 9000 & 0.22 & 5.44 & 6867 & 6.019 & 5.173 & 0.061 \\
\hline 10 & 15.000 & 0.10 & 3.00 & 3600 & 4.263 & 4.028 & 0.051 \\
\hline 11 & 9000 & 0.30 & 7.00 & 3600 & 5.594 & 4.028 & 0.079 \\
\hline 12 & 9000 & 0.10 & 7.00 & 9000 & 5.987 & 5.394 & 0.086 \\
\hline 13 & 12.810 & 0.20 & 7.00 & 3600 & 5.262 & 3.844 & 0.069 \\
\hline 14 & 14.490 & 0.22 & 5.26 & 6668 & 5.591 & 4.825 & 0.052 \\
\hline 15 & 11.400 & 0.10 & 4.34 & 5652 & 5.055 & 4.640 & 0.056 \\
\hline 16 & 12.000 & 0.20 & 6.92 & 8783 & 6.056 & 5.053 & 0.071 \\
\hline 17 & 11.400 & 0.10 & 4.34 & 5652 & 5.081 & 4.372 & 0.077 \\
\hline 18 & 9000 & 0.26 & 3.00 & 3600 & 4.495 & 4.353 & 0.070 \\
\hline 19 & 15.000 & 0.20 & 3.00 & 9000 & 5.290 & 5.179 & 0.055 \\
\hline 20 & 15.000 & 0.10 & 7.00 & 6354 & 5.510 & 4.038 & 0.052 \\
\hline 21 & 12.840 & 0.10 & 4.96 & 9000 & 5.386 & 5.005 & 0.062 \\
\hline 22 & 12.780 & 0.30 & 3.00 & 6192 & 4.885 & 4.788 & 0.106 \\
\hline 23 & 15.000 & 0.30 & 5.08 & 3600 & 4.864 & 3.854 & 0.105 \\
\hline 24 & 9000 & 0.10 & 6.26 & 3600 & 4.653 & 3.918 & 0.067 \\
\hline 25 & 9000 & 0.22 & 5.44 & 6867 & 5.764 & 5.044 & 0.066 \\
\hline
\end{tabular}

\section{Resultados y Discusión}

\section{Cálculo de los pesos de los criterios con Fuzzy AHP}

Para utilizar Fuzzy AHP en evaluar las alternativas se parte del análisis del modelo jerárquico presentado en la Figura 2. Este representa en su nivel superior el objetivo principal o las metas en la solución del problema. En el nivel inferior se encuentran los criterios que son evaluados entre sí y seguidamente con las alternativas. Estos criterios también pueden ser disgregados en sub-criterios. En el último nivel se encontrarán las alternativas que serán seleccionada por nivel de prioridad, y por el resultado de la ponderación que serán fruto cada criterio. 


\section{Figura 2}

Modelo jerárquico para la evaluación de las alternativas

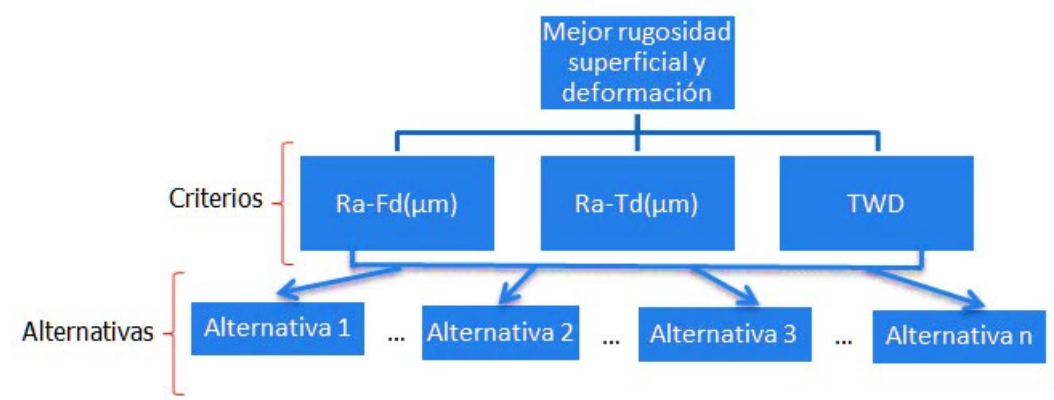

Obtención Matriz difusa por pares para la evaluación de las alternativas. En este paso es importante la evaluación de los expertos a cada criterio y se completan las variables lingüísticas, mediante la asignación directa de una escala, Tabla 4. Finalmente se obtiene la matriz, que se muestra en la Tabla 6.

\section{Tabla 6}

Matriz Difusa por Pares para la Evaluación de las Alternativas

\begin{tabular}{|c|c|c|c|}
\hline & $\operatorname{Ra}-F d(\mu \mathrm{m})$ & $\operatorname{Ra}-T d(\mu \mathrm{m})$ & TWD \\
\hline Ra-Fd( $\boldsymbol{\mu m})$ & $(1,1,1)$ & $(1 / 4,1 / 3,1 / 2)$ & $(1 / 6,1 / 5,1 / 4)$ \\
\hline $\operatorname{Ra}-T d(\mu \mathrm{m})$ & $(2,3,4)$ & $(1,1,1)$ & $(1 / 4,1 / 3,1 / 2)$ \\
\hline TWD & $(4,5,6)$ & $(2,3,4)$ & $(1,1,1)$ \\
\hline
\end{tabular}

Se realizan los cálculos empleando Microsoft Excel para componer la matriz Fuzzy y los valores de los pesos relacionado con los criterios para determinar la mejor alternativa a partir de la jerarquía que establece el método. Los resultados son agrupados en la Tabla 7, se muestra el valor de peso Fuzzy obtenido y su valor centralizado.

\section{Tabla 7}

Resultado del Pesos Fuzzy y el valor centralizado

\begin{tabular}{|c|c|}
\hline $\boldsymbol{w i}$ & Wi Centralizado \\
\hline$(0.0160,0.1462,0.1486)$ & 0.1036 \\
\hline$(0.3279,0.2878,0.2975)$ & 0.3044 \\
\hline$(0.6561,0.5660,0.5538)$ & 0.5920 \\
\hline
\end{tabular}

Los resultados jerárquicos por los pesos se muestran en la Tabla 8.

\section{Tabla 8}

Los pesos y el ranking de los criterios

\begin{tabular}{|c|c|c|}
\hline & Wi & Rank \\
\hline $\operatorname{Ra}-\mathrm{Fd}(\mu \mathrm{m})$ & 0.1036 & 3 \\
\hline $\operatorname{Ra}-\mathrm{Td}(\mu \mathrm{m})$ & 0.3044 & 2 \\
\hline $\mathrm{TWD}$ & 0.5920 & 1 \\
\hline
\end{tabular}




\section{Jerarquía con método COPRAS}

El método COPRAS (Isik, 2016), ayuda a escoger y evaluar la mejor alternativa paso a paso en términos de su importancia, grado de utilidad y decide las soluciones ideales y las peores-ideales. En la solución propuesta, seleccionamos como los atributos de beneficios: RaFd (Rugosidad superficial en dirección del avance), TWD (Deformación de la pieza de estructura delgada) y Ra-Td (Rugosidad superficial en dirección transversal) como no beneficioso. La cantidad de alternativas escogidas es de 25 casos. A partir de los pesos obtenidos por Fuzzy AHP: $\mathrm{W}_{\mathrm{Ra}-\mathrm{Fd}}=0.1036, \mathrm{~W}_{\mathrm{Ra}-\mathrm{Td}}=0.3044$ y $\mathrm{W}_{\mathrm{TWD}}=0.5920$ y teniendo en cuenta que el criterio de mayor peso constituye TWD (Deformación de la pieza de estructura delgada).

Empleando la Ecuación 10, calculamos la matriz de decisión normalizada $\left(\mathrm{X}_{\mathrm{ij}}{ }^{*}\right)$, y para la matriz normalizada por pesos $\left.\left(\mathrm{D}_{\mathrm{ij}}\right)^{*}\right)$ se calcula según la Ecuación 12 , mostrado en la Tabla 9.

\section{Tabla 9}

Cálculo de la Matriz Normalizada por Pesos(Dij)*)

\begin{tabular}{|c|c|c|c|}
\hline No. & Ra-Fd $(\boldsymbol{\mu m})$ & Ra-Td $(\boldsymbol{\mu m})$ & TWD \\
\hline 1 & 0.0035 & 0.0101 & 0.0199 \\
\hline 2 & 0.0042 & 0.0121 & 0.0219 \\
\hline 3 & 0.0051 & 0.0127 & 0.0349 \\
\hline 4 & 0.0044 & 0.0132 & 0.0196 \\
\hline 5 & 0.0042 & 0.0142 & 0.0199 \\
\hline 6 & 0.0041 & 0.0135 & 0.0332 \\
\hline 7 & 0.0040 & 0.0139 & 0.0212 \\
\hline 8 & 0.0045 & 0.0160 & 0.0279 \\
\hline 9 & 0.0047 & 0.0132 & 0.0203 \\
\hline 10 & 0.0033 & 0.0103 & 0.0169 \\
\hline 11 & 0.0043 & 0.0103 & 0.0262 \\
\hline 12 & 0.0046 & 0.0138 & 0.0286 \\
\hline 13 & 0.0041 & 0.0098 & 0.0229 \\
\hline 14 & 0.0043 & 0.0124 & 0.0173 \\
\hline 15 & 0.0039 & 0.0119 & 0.0186 \\
\hline 16 & 0.0047 & 0.0129 & 0.0236 \\
\hline 17 & 0.0039 & 0.0112 & 0.0256 \\
\hline 18 & 0.0035 & 0.0111 & 0.0232 \\
\hline 19 & 0.0041 & 0.0133 & 0.0183 \\
\hline 20 & 0.0043 & 0.0103 & 0.0173 \\
\hline 21 & 0.0042 & 0.0128 & 0.0206 \\
\hline 22 & 0.0038 & 0.0123 & 0.0352 \\
\hline 23 & 0.0038 & 0.0099 & 0.0349 \\
\hline 24 & 0.0036 & 0.0100 & 0.0222 \\
\hline 25 & 0.0045 & 0.0129 & 0.0219 \\
\hline
\end{tabular}

La sumatoria de los valores normalizados ponderados $\left(\mathrm{S}_{(\mathrm{i}+)}\right)$, $\left(\mathrm{S}_{(\mathrm{i}-)}\right)$, el resultado de la importancia relativa de las alternativas $\left(\mathrm{Q}_{\mathrm{i}}\right)$ y el índice de utilidad $\left(\mathrm{U}_{\mathrm{i}}\right)$, que determina la jerarquía de la mejor alternativa de todas las candidatas que permite alcanzar la mejor calidad de rugosidad superficial y la menor desviación lateral de la pieza delgada son calculados con las Ecuaciones 13, 14, 15 y 16 respectivamente y todos los cálculos se muestran en la Tabla 9.

Los parámetros de entrada de la mejor alternativa determinada para la operación de 
fresado a alta velocidad de estructuras delgadas de Al 5083 se muestran en la Tabla 10.

\section{Tabla 10}

Cálculo de $\left(S_{i+}\right),\left(S_{i-}\right),\left(Q_{i}\right)$ y jerarquía COPRAS

\begin{tabular}{|c|c|c|c|c|c|c|c|c|}
\hline No. & $\operatorname{Ra}-F d(\mu m)$ & $\operatorname{Ra}-T d(\mu \mathrm{m})$ & TWD & $\mathbf{S}+\mathbf{i}$ & S-i & Qi & Ui & Rank \\
\hline 1 & 0.0035 & 0.0101 & 0.0199 & 0.0235 & 0.0101 & 0.0236 & 58.8801 & 20 \\
\hline 2 & 0.0042 & 0.0121 & 0.0219 & 0.0261 & 0.0121 & 0.0263 & 65.6078 & 13 \\
\hline 3 & 0.0051 & 0.0127 & 0.0349 & 0.0399 & 0.0127 & 0.0401 & 100.0000 & 1 \\
\hline 4 & 0.0044 & 0.0132 & 0.0196 & 0.024 & 0.0132 & 0.0242 & 60.3543 & 19 \\
\hline 5 & 0.0042 & 0.0142 & 0.0199 & 0.0241 & 0.0142 & 0.0243 & 60.5943 & 18 \\
\hline 6 & 0.0041 & 0.0135 & 0.0332 & 0.0373 & 0.0135 & 0.0375 & 93.6043 & 4 \\
\hline 7 & 0.0040 & 0.0139 & 0.0212 & 0.0253 & 0.0139 & 0.0255 & 63.4714 & 15 \\
\hline 8 & 0.0045 & 0.0160 & 0.0279 & 0.0324 & 0.0160 & 0.0326 & 81.3756 & 6 \\
\hline 9 & 0.0047 & 0.0132 & 0.0203 & 0.0249 & 0.0132 & 0.0251 & 62.5949 & 16 \\
\hline 10 & 0.0033 & 0.0103 & 0.0169 & 0.0202 & 0.0103 & 0.0204 & 50.8205 & 25 \\
\hline 11 & 0.0043 & 0.0103 & 0.0262 & 0.0306 & 0.0103 & 0.0307 & 76.5646 & 7 \\
\hline 12 & 0.0046 & 0.0138 & 0.0286 & 0.0332 & 0.0138 & 0.0334 & 83.2459 & 5 \\
\hline 13 & 0.0041 & 0.0098 & 0.0229 & 0.027 & 0.0098 & 0.0271 & 67.6296 & 10 \\
\hline 14 & 0.0043 & 0.0124 & 0.0173 & 0.0216 & 0.0124 & 0.0218 & 54.2868 & 23 \\
\hline 15 & 0.0039 & 0.0119 & 0.0186 & 0.0225 & 0.0119 & 0.0227 & 56.5454 & 21 \\
\hline 16 & 0.0047 & 0.0129 & 0.0236 & 0.0283 & 0.0129 & 0.0285 & 70.9317 & 9 \\
\hline 17 & 0.0039 & 0.0112 & 0.0256 & 0.0295 & 0.0112 & 0.0297 & 73.9515 & 8 \\
\hline 18 & 0.0035 & 0.0111 & 0.0232 & 0.0267 & 0.0111 & 0.0269 & 67.0249 & 11 \\
\hline 19 & 0.0041 & 0.0133 & 0.0183 & 0.0224 & 0.0133 & 0.0226 & 56.2223 & 22 \\
\hline 20 & 0.0043 & 0.0103 & 0.0173 & 0.0215 & 0.0103 & 0.0217 & 54.0561 & 24 \\
\hline 21 & 0.0042 & 0.0128 & 0.0206 & 0.0248 & 0.0128 & 0.0249 & 62.1849 & 17 \\
\hline 22 & 0.0038 & 0.0123 & 0.0352 & 0.039 & 0.0123 & 0.0392 & 97.6152 & 2 \\
\hline 23 & 0.0038 & 0.0099 & 0.0349 & 0.0386 & 0.0099 & 0.0388 & 96.6587 & 3 \\
\hline 24 & 0.0036 & 0.0100 & 0.0222 & 0.0258 & 0.0100 & 0.0260 & 64.8057 & 14 \\
\hline 25 & 0.0045 & 0.0129 & 0.0219 & 0.0264 & 0.0129 & 0.0266 & 66.2289 & 12 \\
\hline
\end{tabular}

Los parámetros de entrada de la mejor alternativa determinada para la operación de fresado a alta velocidad de estructuras delgadas de Al 5083 se muestran en la Tabla 11.

\section{Tabla 11}

Los Parámetros de Corte de la Mejor Alternativa, para Operación de Fresado a Alta Velocidad de Paredes Delgadas de AL5083

\begin{tabular}{|c|c|c|c|c|c|c|c|}
\hline \multicolumn{4}{|c|}{ Parámetros de corte } & \multicolumn{4}{c|}{ Resultados } \\
\hline No & $\mathbf{S}(\mathbf{r p m})$ & $\mathbf{d o c}(\mathbf{m m})$ & $\mathbf{t s}(\mathbf{m m})$ & $\mathbf{F}(\mathbf{m} / \mathbf{m i n})$ & $\mathbf{R}_{\mathbf{a}}-\mathbf{F}_{\mathbf{d}}(\boldsymbol{\mu m})$ & $\mathbf{R}_{\mathbf{a}}-\mathbf{T}_{\mathbf{d}}(\boldsymbol{\mu \mathbf { m }})$ & $\mathbf{T W D}$ \\
\hline 3 & 15 & 0.3 & 7 & 9000 & 6,5400 & 4.979 & 0.105 \\
\hline
\end{tabular}

\section{Conclusiones}

Cada día en los centros de maquinado se hace más importante la evaluación y correcta selección de los parámetros para realizar los procesos de maquinado a alta velocidad. La elección de la mejor alternativa de manera rápida, prevalece en los criterios de los empresarios e ingenieros para mantener la calidad y bajos costos de las producciones. 
En la evaluación de los criterios el método Fuzzy-AHP evalua que, dentro de la solución óptima, el criterio de deformación de la pieza de estructura delgada (TWD), es el de mayor importancia con valor: $\mathrm{W}_{\mathrm{TWD}}=0.5920$. A partir de lo anterior al aplicar COPRAS y calcular el índice de utilidad $\left(\mathrm{U}_{\mathrm{i}}\right)$ se determina que la alternativa 3 ofrece con los parámetros de entrada $S=15 \mathrm{rpm}$, doc $=0.3 \mathrm{~mm}$, ts $=7.0 \mathrm{~mm}$, la garantía de los resultados para una mejor calidad y sin deformar la pieza.

El trabajo de investigación demuestra que al emplear el método de inteligencia artificial Fuzzy-AHP y el multicriterio COPRAS como una herramienta de evaluación clara, objetiva y confiable, se logra el objetivo de su solución que es determinar la jerarquía de las alternativas analizadas para la toma de decisiones. Todo esto basado en los mejores factores que tienen mayor impacto para los procesos de fabricación a altas velocidades en piezas de estructuras delgadas y como material las aleaciones de aluminio AL 5083.

Como recomendación la investigación propone que el resultado de este trabajo se extienda en los talleres de maquinado para mejorar la toma de decisiones. Además, realizar las pruebas del mismo en piezas de estructura delgadas con material de alta dureza o aceros especiales.

\section{Referencias}

Bhowmik, S., Jagadish, \& Gupta, K. (2019). Modeling and Optimization of Advanced Manufacturing Processes. https://doi.org/10.1007/978-3-030-00036-3.

Ighravwe, D. E., \& Oke, S. A. (2020). A two-stage fuzzy multi-criteria approach for proactive maintenance strategy selection for manufacturing systems. SN Applied Sciences, 2(10), 1683. https://doi.org/10.1007/s42452-020-03484-6

Isik, N. K. a. A. (2016). Integration of Macbeth and copras methods to select air compressor for a textile company. Decision Science Letters, 5, 381-394, Article 3. https://doi.org/ http://dx.doi.org/10.5267/j.dsl.2016.2.003.

Jasiulewicz-Kaczmarek, M., Antosz, K., Wyczółkowski, R., Mazurkiewicz, D., Sun, B., Qian, C., \& Ren, Y. (2021). Application of MICMAC, Fuzzy AHP, and Fuzzy TOPSIS for Evaluation of the Maintenance Factors Affecting Sustainable Manufacturing. Energies, 14(5), 1436. https://doi.org/10.3390/en14051436.

Kaori Ota, N. T., Hayao Miyagi (2008). Group Decision-Making Model in Fuzzy AHP Based on the Variable Axis Method. In T. I. o. E. E. o. Japan (Ed.), (pp. 6).

Keshavarz-Ghorabaee, M., Govindan, K., Amiri, M., Zavadskas, E. K., \& Antuchevičienė, J. (2019). An integrated type-2 fuzzy decision model based on WASPAS and SECA for evaluation of sustainable manufacturing strategies. Journal of Environmental Engineering and Landscape Management, 27(4), 187-200. https://doi.org/10.3846/jeelm.2019.11367.

Mehdi Ajalli, H. A., Abdolkarim Mohammadi Balani, Mahdi Rezaei. (2017). Application of Fuzzy AHP and COPRAS to Solve the Supplier Selection Problems. International Journal of Supply Chain Management, 6, 8 .

Paker, F. A., Alppay, C., \& Sertyeşilişik, B. (2018). Use of the AHP Methodology in Vehicle Design Process Dynamics: Determination of the Most Effective Concept Phases for the New Automotive Product. Journal of Transportation Technologies, 08(04), 312-330. https://doi.org/10.4236/jtts.2018.84017.

Patil, R. B., \& Kothavale, B. S. (2020). Criticality Analysis of CNC Turning Center Using Analytic Hierarchy Process. In Reliability and Risk Assessment in Engineering (pp. 61-76). https://doi.org/10.1007/978981-15-3746-2_6 
Sen, B., Hussain, S. A. I., Gupta, A. D., Gupta, M. K., Pimenov, D. Y., \& Mikołajczyk, T. (2020). Application of Type-2 Fuzzy AHP-ARAS for Selecting Optimal WEDM Parameters. Metals, 11(1), 42. https://doi.org/10.3390/met11010042

Singh, R. P., Tyagi, M., \& Kataria, R. (2019). Selection of the Optimum Hole Quality Conditions in Manufacturing Environment Using MCDM Approach: A Case Study. In Operations Management and Systems Engineering (pp. 133-152). https://doi.org/10.1007/978-981-13-6476-1_8

Sivam Sundarlingam Paramasivam, S. S., Kumaran, D., Loganathan, G. B., Saravanan, K., Rajendran, R., \& Sriram, H. (2019). Development and Influence of Setting Process Variables in Single Point Incremental Sheet Metal Forming of AA 8011 Using Complex Proportional Assessment and ANOVA. SAE Technical Paper Series,

Sofuoğlu, M. A. A new hybrid decision-making strategy of cutting fluid selection for manufacturing environment. Sādhanā, 46(2), 94. https://doi.org/10.1007/s12046-021-01618-z

Turskis, E. K. Z. a. Z. (2008). A new logarithmic normalization method in games theory. Informática, 19, 12.

Yadav, A., \& Jayswal, S. C. (2018). Modelling of flexible manufacturing system: a review. International Journal of Production Research, 56(7), 1-24. https://doi.org/10.1080/00207543.2017.1387302

Yadav, G., Seth, D., \& Desai, T. N. (2018). Application of hybrid framework to facilitate lean six sigma implementation: a manufacturing company case experience. Production Planning \& Control, 29(3), 117. https://doi.org/10.1080/09537287.2017.1402134 